Results: 542 specimens were processed. There were 16 Campylobacter, 7 Salmonella and 2 non-dysenteriae Shigella spp. positive by culture and 23 Campylobacter, 9 Salmonella and 3 non-dysenteriae Shigella spp. positive by PCR. All culture positive specimens were PCR positive. Sensitivity of PCR was $100 \%$ and specificity 99\%, with an NPV of $100 \%$ for Campylobacter, Salmonella and non-dysenteriae Shigella spp. PPV was $78 \%$ for Salmonella spp., $70 \%$ for Campylobacter spp. and $67 \%$ for non-dysenteriae Shigella spp.

Discussion: The PCR allows considerably faster turn-around time with high sensitivity and specificity. Published data, discrepancy resolution and clinical correlation suggests this low PPV reflects a proportion of PCR-positive but culture negative true disease.

\section{FIRST STEPS IN THE HARMONISATION OF ADULT PLASMA AMINO ACID REFERENCE INTERVALS IN AUSTRALASIA}

Mark de Hora ${ }^{1}$, James Pitt ${ }^{2}$, Avantika Mishra ${ }^{2}$,

Beena Devanapalli ${ }^{3}$, Brett McWhinney ${ }^{4}$, Avis McWhinney ${ }^{5}$, Lawrence Greed $^{6}$, Kevin Carpenter ${ }^{3}$

${ }^{1}$ Chemical Pathology, LabPlus, Auckland, New Zealand;

${ }^{2}$ Victoria Biochemical Genetics, ${ }^{3}$ NSW Biochemical Genetics,

${ }^{4}$ Queensland Pathology, ${ }^{5}$ Mater Health Service, and ${ }^{6}$ WA

Biochemical Genetics, Australia

Six laboratories in Australasia use the same technology to measure plasma amino acids (PLAA) to aid in the investigation of associated inborn errors of metabolism (IEM). There is considerable variation in reference intervals (RI) for PLAA and harmonisation across the laboratory group was suggested.

Aim: To determine adult PLAA RIs using combined data from the six Australasian laboratories.

Method: Inter-laboratory variation was determined for PLAA by analysing External Quality Assurance (EQA) $(n=8)$ returns from each laboratory.

Results of 22 PLAA on 2107 patients over 16 years of age from 6 centres were statistically analysed after removal of known IEM patients and outliers. Gender specific data and population distributions were analysed.

Results: The between laboratory coefficient of variation (CV) for 17 amino acids was $4-10 \%$ and 4 amino acids had CVs $>15 \%$. There was no significant bias from the ERNDIM group. There were gender specific interval differences for 11 amino acids; 2 were normally distributed; 14 required log-transformation and 6 were calculated non-parametrically.

Conclusions: There is acceptable agreement for most amino acids but significant differences for some amino acids possibly due variation in standardisation and specimen handling.

Harmonisation is feasible after agreed changes to standard procedures in laboratories.

\section{WHAT'S THE EMERGENCY? IDENTIFICATION OF IRON DEFICIENCY ANAEMIA TO EVALUATE TRANSFUSION APPROPRIATENESS}

Nora Lee, Terri Dunstan, Rebecca Davidson, Christine Michael, Erica Wood, Richard King

Monash Health, Vic, Australia
Aim: We evaluated anaemia management and transfusion practice in the emergency department (ED) as part of a comprehensive audit program in place for compliance with NSQHS Standard 7 and NHMRC Patient Blood Management guidelines.

Methods: A pilot audit of anaemia presentations was conducted over one month. Patients were selected from blood bank records indicating transfusion of red cells. Iron status was categorised as 'deficient', 'probable', 'unlikely' or 'uncertain' using FBE, blood film and iron studies, where performed. Red cell transfusion was 'appropriate', 'appropriate indication (not quantity)' or 'possibly not required' using clinical and laboratory findings. Results: Of 31 cases (17 acute bleeders, 11 no acute bleeding, 3 with transfusion decisions made beyond ED), only $55 \%$ had iron studies, of which $25 \%$ were iron-deficient and replaced with iron. $23 \%$ had probable iron deficiency. In subacute anaemia, $82 \%$ had iron studies, of which $78 \%$ were both iron-deficient and treated with iron. $11 \%$ of transfusions were possibly not required, $31 \%$ appropriate and $58 \%$ overtransfused.

Conclusion: Identification, investigation and management of iron deficiency can be improved in ED presentations with anaemia and could avoid transfusion in some patients. This audit establishes new baseline indicators upon which we can better align our PBM practice with guidelines.

\section{UNUSUAL CAUSE OF PSEUDOHYPERKALAEMIA IN PRIMARY CARE CLINIC}

Vanessa Man Har Lo Clinical Pathology Laboratory, Department of Pathology, Hong Kong Sanatorium and Hospital, Hong Kong Special Administrative Region

Backgrounds: Our hospital has three primary care clinics (PCC1, 2 and 3) outside the main campus. Blood sampled in PCC were sent to the hospital laboratory for analysis with transportation time 2-6 hours. PCC1 accounted for the highest rate of pseudohyperkalaemia, $21 \%$, while the rest were below $5 \%$. Root cause identification followed by constructive solution is urgently required to avoid unnecessary and potentially detrimental therapy due to pseudohyperkalaemia.

Method: From 3 October to 28 November 2013 systematic root cause analysis was conducted by simulation study focussing on preanalytical procedures including: (1) blood sampling technique, (2) sample preparation procedure while sitting in PCC waiting for courier to pick up, (3) transportation conditions including temperature and turbulence effect leading to red cell sediments remixed with plasma during speedy driving, and (4) potassium stability in gel separated plasma up to 9 hours. Statistical analysis tools Deming regression and Bland and Altman plot were adopted.

Results: Excessive fist clenching was identified to be the culprit. Pseudohyperkalaemia was recorded in 10 out of 16 study cases. Upon elimination of the inappropriate procedure, up to 22 September 2015 the occurrence rate of pseudohyperkalaemia in PCC1 dropped dramatically to $7 \%$, comparable with those in PCC2 and PCC3. 